Unspecified Journal

Volume 00, Number 0, Pages 000-000

$\mathrm{S}$ ????-????(XX)0000-0

\title{
SOME QUESTIONS ABOUT SEMISIMPLE LIE GROUPS ORIGINATING IN MATRIX THEORY
}

\author{
DRAGOMIR Ž. ĐOKOVIĆ AND TIN-YAU TAM
}

\begin{abstract}
We generalize the well-known result that a square traceless complex matrix is unitarily similar to a matrix with zero diagonal to arbitrary semisimple complex Lie groups $G$ and their Lie algebras $\mathfrak{g}$ under the action of a maximal compact subgroup $K$ of $G$. We also introduce a natural partial order on $\mathfrak{g}: x \leq y$ if $f(K \cdot x) \subseteq f(K \cdot y)$ for all $f \in \mathfrak{g}^{*}$. This partial order is $K$-invariant and induces a partial order on the orbit space $\mathfrak{g} / K$. We prove that, under some restrictions on $\mathfrak{g}$, the set $f(K \cdot x)$ is star-shaped with respect to the origin.
\end{abstract}

\section{INTRODUCTION}

In this paper we consider some interesting well known facts from Matrix Theory and try to generalize them to arbitrary semisimple complex Lie groups. For instance, it is known that every $n$ by $n$ complex matrix $x$ with zero trace is unitarily similar to a matrix with zero diagonal. We can view $x$ as an element of the Lie algebra $\mathfrak{g}=\mathfrak{s l}(n, \mathbf{C})$ of the group $G=\operatorname{SL}(n, \mathbf{C})$ and the special unitary group $K=\mathrm{SU}(n)$ as a maximal compact subgroup of $G$. The diagonal matrices in $\mathfrak{g}$ form a Cartan subalgebra $\mathfrak{h}$ of $\mathfrak{g}$, and those with purely imaginary diagonal entries form a Cartan subalgebra $\mathfrak{t}$ of the Lie algebra $\mathfrak{k}=\mathfrak{s u}(n)$ of $K$. The subspace of $\mathfrak{g}$ consisting of matrices with zero diagonal is just the sum of all root spaces of $(\mathfrak{g}, \mathfrak{h})$. Two matrices $x, y \in \mathfrak{g}$ are unitarily similar if and only if they belong to the same orbit of $K$ under the restriction of the adjoint representation of $G$ to $K$.

We show (see Theorem 3.4) that this matrix result continues to hold in general when $G$ is an arbitrary semisimple complex Lie group, $K$ a maximal compact subgroup of $G$, and $\mathfrak{h}$ a Cartan subalgebra of $\mathfrak{g}$ which is obtained as the complexification of a Cartan subalgebra $\mathfrak{t}$ of $\mathfrak{k}$. Then it says that every $K$-orbit, say $K \cdot x$, in $\mathfrak{g}$ meets the sum of all root spaces of $(\mathfrak{g}, \mathfrak{h})$, which is also the orthogonal complement of $\mathfrak{h}$ in $\mathfrak{g}$ with respect to the Killing form of $\mathfrak{g}$.

As a real $K$-module, $\mathfrak{g}$ is just the direct sum of two copies of the adjoint module $\mathfrak{k}$ of $K: \mathfrak{g}=\mathfrak{k} \oplus i \mathfrak{k}$. While the orbit space $\mathfrak{k} / K$ is homeomorphic to a closed Weyl chamber in $\mathfrak{t}$, we do not know in general the description of the orbit space $\mathfrak{g} / K$. For the special case $G=\mathrm{SL}(2, \mathbf{C})$ see Proposition 4.2.

We also introduce an interesting partial order " $\leq$ " in $\mathfrak{g}$ : We say that $x \leq y$ $(x, y \in \mathfrak{g})$ if $f(K \cdot x) \subseteq f(K \cdot y)$ for all complex linear functionals $f \in \mathfrak{g}^{*}$. This order is compatible with the action of $K$ and so it induces a partial order on the orbit space $\mathfrak{g} / K$. One of us has conjectured that the image $f(K \cdot x)$ contains the

1991 Mathematics Subject Classification. Primary 05B15, 05B20; Secondary 05B05.

The first author was supported in part by the NSERC Grant A-5285.

(C)0000 (copyright holder) 
origin and is star-shaped with respect to the origin (see Conjecture 3.8). We prove (see Theorem 3.11) that this conjecture is valid if $\mathfrak{g}$ is simply laced and has no components of type $E_{8}$.

We denote by $W$ the Weyl group of $(\mathfrak{g}, \mathfrak{h})$ and by $\mathfrak{b}$ a Borel subalgebra of $\mathfrak{g}$ containing $\mathfrak{h}$. The readers may be interested in the following open questions:

(1) Are the assertions of Proposition 3.12 valid without any restrictions on $\mathfrak{g}$ ?

(2) Is the Conjecture 3.8 true in general?

(3) Find a simple test for $x \leq y$ to hold for arbitrary $x, y \in \mathfrak{g}$ when $\mathfrak{g}=\mathfrak{s l}(2, \mathbf{C})$, or (more ambitiously) for arbitrary semisimple $\mathfrak{g}$

(4) If $O$ is the orthogonal (with respect to the Killing form) projection of $\mathfrak{b} \cap K \cdot x$ to $\mathfrak{h}$, is it true that $O$ is $W$-stable (or even a single $W$-orbit)?

(5) Describe the homeomorphism type of the orbit space $\mathfrak{g} / K$.

\section{Preliminaries}

Let $K$ be a connected compact semisimple Lie group, $G$ its complexification, and let $\mathfrak{k}$ and $\mathfrak{g}$ be their respective Lie algebras. Thus $\mathfrak{g}=\mathfrak{k} \oplus i \mathfrak{k}$. We fix a maximal torus $T$ of $K$ and denote its Lie algebra by $\mathfrak{t}$. Then $\mathfrak{h}=\mathfrak{t} \oplus i \mathfrak{t}$ is a Cartan subalgebra of $\mathfrak{g}$. Let $H$ denote the (algebraic) maximal torus of $G$ with Lie algebra $\mathfrak{h}$.

Let $l$ be the rank of $G$, i.e., $l=\operatorname{dim}_{\mathbf{C}}(\mathfrak{h})$. By $\mathfrak{g}^{*}$ we denote the dual of $\mathfrak{g}$ (as a complex vector space).

Let $R$ be the root system of $(\mathfrak{g}, \mathfrak{h})$ and $\Pi$ a fixed base of $R$. The set of positive roots (with respect to $\Pi$ ) is denoted by $R^{+}$. As usual, $\mathfrak{g}^{\alpha}$ denotes the root space of a root $\alpha$. We introduce the maximal nilpotent subalgebras $\mathfrak{n}$ and $\mathfrak{n}^{-}$of $\mathfrak{g}$ :

$$
\mathfrak{n}=\sum_{\alpha \in R^{+}} \mathfrak{g}^{\alpha}, \quad \mathfrak{n}^{-}=\sum_{\alpha \in R^{+}} \mathfrak{g}^{-\alpha} .
$$

Then $\mathfrak{b}=\mathfrak{h}+\mathfrak{n}$ is a Borel subalgebra of $\mathfrak{g}$, and let $B$ be the corresponding Borel subgroup of $G$. The coroot corresponding to a root $\alpha$ is denoted by $H_{\alpha}$. Recall that $\left[\mathfrak{g}^{\alpha}, \mathfrak{g}^{-\alpha}\right]$ is a 1-dimensional subspace of $\mathfrak{h}$ and $H_{\alpha}$ is the unique element of $\left[\mathfrak{g}^{\alpha}, \mathfrak{g}^{-\alpha}\right]$ such that $\alpha\left(H_{\alpha}\right)=2$. (For more details see eg. [2, Chapitre $8, \S 2$, Théorème 1].) The Weyl group of $(\mathfrak{g}, \mathfrak{h})$ will be denoted by $W$.

We denote by $\theta$ the Cartan involution of $\mathfrak{g}$ (when viewed as a real Lie algebra): It is identity on $\mathfrak{k}$ and negative identity on $i \mathfrak{k}$. It can be lifted to an anti-holomorphic involutorial automorphism of $G$, which we also denote by $\theta$. Then $K=G^{\theta}$, i.e., $K$ is the set of $\theta$-fixed points of $G$. We remark that $\theta(\mathfrak{h})=\mathfrak{h}$ and $\theta\left(\mathfrak{g}^{\alpha}\right)=\mathfrak{g}^{-\alpha}$ for all $\alpha \in R$.

The Killing form of $\mathfrak{g}$ will be denoted by $\varphi$. Unless stated otherwise, the orthogonal complements will be taken with respect to $\varphi$. As $\varphi$ is nondegenerate, it induces a vector space isomorphism $\mathfrak{g} \rightarrow \mathfrak{g}^{*}$ sending $x \rightarrow \varphi_{x}$ where $\varphi_{x}(y)=\varphi(x, y)$ for all $y \in \mathfrak{g}$.

Definition 2.1. An element $x \in \mathfrak{g}$ is nilpotent (resp. semisimple) if the linear operator $\operatorname{ad}(x): \mathfrak{g} \rightarrow \mathfrak{g}$ has the same property. An element $f=\varphi_{x} \in \mathfrak{g}^{*}$ is nilpotent (resp. semisimple) if $x$ has the same property.

We shall consider the adjoint action, Ad, of $G$ on $\mathfrak{g}$ and its restriction to $K$. We write $a \cdot x$ instead of $\operatorname{Ad}(a)(x)$ for $a \in G$ and $x \in \mathfrak{g}$. The co-adjoint action of $G$ on $\mathfrak{g}^{*}$ is defined by $a \cdot f=f \circ \operatorname{Ad}\left(a^{-1}\right)$, where $a \in G$ and $f \in \mathfrak{g}^{*}$. Thus we have $(a \cdot f)(x)=f\left(a^{-1} \cdot x\right)$ for $a \in G, f \in \mathfrak{g}^{*}$, and $x \in \mathfrak{g}$. 
We refer to the minimal ideals of $\mathfrak{g}$ as its components. A subset $F$ of $\mathfrak{g}$ is starshaped with respect to the origin if $x \in F$ and $t \in[0,1)$ imply that $t x \in F$.

\section{The ACTION OF $K$ ON $\mathfrak{g}$}

It is well known that $\mathfrak{b}$ meets every $G$-orbit in $\mathfrak{g}$ (see eg. [6, Section 16]), which may be viewed as a generalization of the Jordan canonical form of an $n$ by $n$ complex matrix. This is also true for the $K$-orbits in $\mathfrak{g}$. It generalizes Schur triangularization theorem which asserts that each $n$ by $n$ complex matrix is unitarily similar to an upper triangular matrix.

Proposition 3.1. $\mathfrak{b}$ meets every $K$-orbit in $\mathfrak{g}$.

Proof. This follows from the result just mentioned and the well known fact that $G=K B=B K$, which is a consequence of the global Iwasawa decomposition (see eg. [4, Chapter VI, Theorem 6.3]).

We remark that Schur triangularization theorem also asserts that the diagonal elements in an upper triangular form of the $n$ by $n$ complex matrix $x$, i.e., the eigenvalues of $x$, can be arranged in any order. Thus we ask Question (4) in the Introduction.

In Matrix Theory, the following result is well known (see eg. [5, Theorem 1.3.4]):

Proposition 3.2. Every $n$ by $n$ complex matrix of trace 0 is unitarily similar to a matrix with zero diagonal.

In order to extend this result to complex semisimple Lie algebras, we need the following lemma. But first let us recall a few definitions that we will need. A connected Lie group is called almost simple if its Lie algebra is simple, and the quotient of a direct product of Lie groups by a discrete central subgroup is called an almost direct product. A root subsystem $R_{1}$ of a root system $R$ is said to be closed if $\alpha, \beta \in R_{1}$ and $\alpha+\beta \in R$ imply that $\alpha+\beta \in R_{1}$.

Lemma 3.3. There exists a closed connected $\theta$-stable complex semisimple Lie subgroup $S$ of $G$ containing $H$ and such that $S$ is an almost direct product of $\theta$-stable almost simple subgroups $S_{i}(i=1, \ldots, m)$ of type $A$.

Proof. Without any loss of generality, we may assume that $\mathfrak{g}$ is simple, and not of type $A_{l}$. We remark that if $R_{1}$ is a closed root subsystem of $R$, then the corresponding semisimple subalgebra $\mathfrak{g}_{1}$ of $\mathfrak{g}$ is $\theta$-stable.

If $-1 \in W$ then there exists a set of $l$ strongly orthogonal roots in $R$, say $\left\{\beta_{1}, \ldots, \beta_{l}\right\}$ [1, Chapitre VI, $\S 1$, Exercice 15]. We set $m=l$ and take $\mathfrak{s}_{i}=\mathfrak{g}^{\beta_{i}}+$ $\mathfrak{g}^{-\beta_{i}}+\mathbf{C} H_{\beta_{i}}$ for $1 \leq i \leq l$. In this case each $S_{i}$ is of type $A_{1}$.

Next assume that $\mathfrak{g}$ is of type $D_{l}$, with $l$ odd. As $R$ has a closed root subsystem of type $A_{l}$, we can take $S$ to be the corresponding subgroup of type $A_{l}$.

Finally, if $\mathfrak{g}$ is of type $E_{6}$, then $R$ has a closed root subsystem $\mathfrak{s}$ of type $3 A_{2}$. The corresponding subgroup $S$ is an almost direct product $S=S_{1} S_{2} S_{3}$, where each $S_{i}$ is of type $A_{2}$.

We have exhausted all possibilities (see [1, Planches I-IX]).

Note that $\mathfrak{h}^{\perp}=\mathfrak{n}+\mathfrak{n}^{-}$. The following theorem generalizes the above matrix result to our setting.

Theorem 3.4. $\mathfrak{h}^{\perp}$ meets every $K$-orbit in $\mathfrak{g}$. 
Proof. Let $S=S_{1} S_{2} \cdots S_{m}$ be as in Lemma 3.3. Then $\mathfrak{h}_{i}=\mathfrak{s}_{i} \cap \mathfrak{h}$ is a Cartan subalgebra of $\mathfrak{s}_{i}$, and $\mathfrak{h}$ is a direct sum of the $\mathfrak{h}_{i}$. The algebra $\mathfrak{s}$ is a direct sum of its ideals $\mathfrak{s}_{i}$. Denote by $\mathfrak{q}$ the sum of the root spaces $\mathfrak{g}^{\alpha}$ that are not contained in $\mathfrak{s}$. Then $\mathfrak{g}=\mathfrak{s} \oplus \mathfrak{q}, \mathfrak{q} \subseteq \mathfrak{h}^{\perp}$, and $\mathfrak{q}$ is $S$-stable. The subgroup $K_{i}=K \cap S_{i}$ is a maximal compact subgroup of $S_{i}$.

An arbitrary $x \in \mathfrak{g}$ can be decomposed uniquely as

$$
x=\sum_{i=1}^{m} x_{i}+x^{\prime}
$$

where $x_{i} \in \mathfrak{s}_{i}$ and $x^{\prime} \in \mathfrak{q}$. Since each $S_{i}$ is of type $A$, Proposition 3.2 implies that there exists $a_{i} \in K_{i}$ such that $a_{i} \cdot x_{i} \in \mathfrak{h}^{\perp} \cap \mathfrak{s}_{i}$. If $a=a_{1} a_{2} \cdots a_{m}$, then $a \in K \cap S$ and

$$
a \cdot x=\sum_{i=1}^{m} a_{i} \cdot x_{i}+a \cdot x^{\prime} \in \sum_{i=1}^{m} \mathfrak{h}^{\perp} \cap \mathfrak{s}_{i}+\mathfrak{q} \subseteq \mathfrak{h}^{\perp} .
$$

Let us illustrate this theorem by a concrete matrix example which does not seem to be known.

Example 3.5. Let $x$ be an $n$ by $n$ skew-symmetric complex matrix. Then there exists a real orthogonal matrix $a$ such that the matrix $y=a x a^{-1}$ has the 2 by 2 diagonal blocks along the diagonal corresponding to the partition $\{1,2\},\{3,4\}, \ldots$ all zero. This is obtained from Theorem 3.4 by taking $G=\mathrm{SO}(n, \mathbf{C}), K=\mathrm{SO}(n)$, $\mathfrak{g}$ to be the Lie algebra of all $n$ by $n$ skew-symmetric complex matrices, and $\mathfrak{h}$ to be the Cartan subalgebra [4, p. 187-189] consisting of block-diagonal matrices with the diagonal blocks of size 2 corresponding to the above partition (except, when $n$ is odd, the last block is of size 1).

Our next objective is to introduce a partial order on $\mathfrak{g}$ (which depends on our choice of $K$, a maximal compact subgroup of $G$ ).

Proposition 3.6. For $x, y \in \mathfrak{g}$ the following are equivalent:

(i) $f(K \cdot x) \subseteq f(K \cdot y), \forall f \in \mathfrak{g}^{*}$,

(ii) $f(x) \in f(K \cdot y), \forall f \in \mathfrak{g}^{*}$.

Proof. It is obvious that (i) implies (ii). Assume that (ii) holds. Let $a \in K$ and $f \in \mathfrak{g}^{*}$ be arbitrary. As $f \circ \operatorname{Ad}(a) \in \mathfrak{g}^{*}$, the hypothesis gives:

$$
f(a \cdot x)=f \circ \operatorname{Ad}(a)(x) \in f \circ \operatorname{Ad}(a)(K \cdot y)=f(a K \cdot y)=f(K \cdot y) .
$$

Hence (i) holds.

Definition 3.7. For $x, y \in \mathfrak{g}$ we write $x \leq y$ if the two conditions of the above proposition are satisfied.

Clearly, the relation " $\leq$ " defines a partial order on $\mathfrak{g}$. This partial order is strongly $K$-invariant in the sense that $x \leq y$ implies that $a \cdot x \leq b \cdot y$ for $a, b \in K$, and so it induces a partial order on the orbit space $\mathfrak{g} / K$. A more transparent description of this important partial order is lacking. We shall take a closer look at the special case $\mathfrak{g}=\mathfrak{s l}_{2}$ in the next section.

Recently Cheung and Tsing [3] proved that if $\mathfrak{g}$ is of type $A_{l}$ then for every $x \in \mathfrak{g}$ and every $f \in \mathfrak{g}^{*}$ the set $f(K \cdot x)$ is star-shaped with respect to the origin. In particular, $0 \in f(K \cdot x)$, i.e., $\operatorname{ker}(f)$ meets $K \cdot x$. Then the second author conjectured that the result of Cheung and Tsing is valid in the general case. 
Conjecture 3.8. [11] For $x \in \mathfrak{g}$ and $f \in \mathfrak{g}^{*}$, the set $f(K \cdot x)$ is star-shaped with respect to the origin.

This conjecture can be reformulated in terms of the partial order " $\leq$ ". The statement is independent of the choice of $\mathfrak{k}$ since the maximal compact subgroup $K$ of $G$ is unique up to an inner automorphism of $G$ [4, p.256].

Conjecture 3.9. [11] For $x \in \mathfrak{g}$ and $t \in[0,1]$, we have $t x \leq x$.

It is not hard to reduce the proof of this conjecture to the case of simple Lie algebras. We do this in the next lemma.

Lemma 3.10. Assume that $\mathfrak{k}$ is a direct sum of two nonzero ideals $\mathfrak{k}=\mathfrak{k}_{1} \oplus \mathfrak{k}_{2}$ and let $K=K_{1} K_{2}$ be the corresponding (almost direct) decomposition of $K$. Then $\mathfrak{g}$ is $a$ direct sum of the ideals $\mathfrak{g}_{1}=\mathfrak{k}_{1}+i \mathfrak{k}_{1}$ and $\mathfrak{g}_{2}=\mathfrak{k}_{2}+i \mathfrak{k}_{2}$. Let $x, y \in \mathfrak{g}$ be decomposed as $x=x_{1}+x_{2}, y=y_{1}+y_{2}$ with $x_{1}, y_{1} \in \mathfrak{g}_{1}$ and $x_{2}, y_{2} \in \mathfrak{g}_{2}$. Then $x_{1} \leq y_{1}$ in $\mathfrak{g}_{1}$ and $x_{2} \leq y_{2}$ in $\mathfrak{g}_{2}$ if and only if $x \leq y$ in $\mathfrak{g}$.

Proof. We have $K \cdot x=K_{1} \cdot x_{1}+K_{2} \cdot x_{2}$ and $K \cdot y=K_{1} \cdot y_{1}+K_{2} \cdot y_{2}$. Hence $K \cdot x \subseteq K \cdot y$ if and only if $K_{1} \cdot x_{1} \subseteq K_{1} \cdot y_{1}$ and $K_{2} \cdot x_{2} \subseteq K_{2} \cdot y_{2}$.

We say that $\mathfrak{g}$ is simply laced if the simple components of $\mathfrak{g}$ are of type $A, D$, or $E$. We can prove that the above conjecture is true if $\mathfrak{g}$ is simply laced and has no components of type $E_{8}$.

Theorem 3.11. If $\mathfrak{g}$ is simply laced and has no components of type $E_{8}$, then Conjecture 3.8 is valid.

Proof. In view of Lemma 3.10 we may assume that $\mathfrak{g}$ is simple. If $\mathfrak{g}$ is of type $A_{l}$ then the conjecture holds by the result of Cheung and Tsing [3].

Let $x \in \mathfrak{g}$ be arbitrary. We have to show that $t x \leq x$ for $t \in[0,1)$. Since the partial order " $\leq$ " is $K$-invariant, by Theorem 3.4 we may assume that $x \in \mathfrak{h}^{\perp}$, i.e.,

$$
x=\sum_{\alpha \in R} x_{\alpha}, \quad x_{\alpha} \in \mathfrak{g}^{\alpha} .
$$

Assume first that $\mathfrak{g}$ is of type $D_{l}$. In this proof we assume that $\mathfrak{g}$ is realized as a Lie algebra of linear operators on a complex vector space $V$ of dimension $2 l$ as in [2, Chapitre VIII, $\S 13$, No. 4]. We shall make use of the basis $\left\{H_{1}, H_{2}, \ldots, H_{l}\right\}$ of $\mathfrak{h}$ defined there, and its dual basis $\left\{\varepsilon_{1}, \varepsilon_{2}, \ldots, \varepsilon_{l}\right\}$ of $\mathfrak{h}^{*}$. We recall that $R=$ $\left\{ \pm \varepsilon_{i} \pm \varepsilon_{j}: 1 \leq i<j \leq l\right\}$ where all four sign combinations should be taken. Observe that each $H_{m} \in i$ t because all roots take real values on $H_{m}$.

Each $m \in\{1,2, \ldots, l\}$ determines a partition $R=R_{m}(-1) \cup R_{m}(0) \cup R_{m}(1)$, where

$$
R_{m}(k)=\left\{\alpha \in R: \alpha\left(H_{m}\right)=k\right\}, \quad k \in\{0, \pm 1\} .
$$

The subset $R_{m}(0)$ is a closed root subsystem of $R$ of type $D_{l-1}$, and each of the subsets $R_{m}( \pm 1)$ has cardinality $2(l-1)$.

Let $L_{m}$ be the linear operator on $\mathfrak{g}$ which fixes the elements of $\mathfrak{h}$ and those of the root spaces $\mathfrak{g}^{\alpha}$ for $\alpha \in R_{m}(0)$ and on the other root spaces acts as multiplication by the scalar $\sqrt{t}$. Thus we have

$$
L_{m}(x)=\sum_{\alpha \in R_{m}(0)} x_{\alpha}+\sqrt{t} \sum_{\alpha \in R_{m}( \pm 1)} x_{\alpha} .
$$

We claim that $L_{m}(x) \leq x$. To prove this claim, let $f \in \mathfrak{g}^{*}$ be arbitrary and we have to show that $f\left(L_{m}(x)\right) \in f(K \cdot x)$. Let $s$ be a real parameter. Since $H_{m} \in i \mathfrak{t}$, 
we have $i s H_{m} \in \mathfrak{t}$, and so $\exp \left(i s H_{m}\right) \in K$. Moreover this element sends $x_{\alpha}$ to $e^{i s \alpha\left(H_{m}\right)} x_{\alpha}$. Consequently

$$
f\left(\exp \left(i s H_{m}\right) \cdot x\right)=a+b e^{i s}+c e^{-i s},
$$

where

$$
a=f\left(\sum_{\alpha \in R_{m}(0)} x_{\alpha}\right), \quad b=f\left(\sum_{\alpha \in R_{m}(1)} x_{\alpha}\right), \quad c=f\left(\sum_{\alpha \in R_{m}(-1)} x_{\alpha}\right) .
$$

We now make use of an argument from [3]. As $s$ varies, the point $a+b e^{i s}+c e^{-i s}$ traces an ellipse $\mathcal{E}$ in the complex plane, with $a$ as its center. If $|b|=|c|$ the ellipse degenerates to a line segment or just a point. Since $t \in[0,1)$, the point $f\left(L_{m}(x)\right)=a+\sqrt{t}(b+c)$ lies inside $\mathcal{E}$, or on $\mathcal{E}$ in the degenerate case. Clearly, we can dismiss the degenerate case.

Let $y \in \mathfrak{g}$ be such that $f=\varphi_{y}$. By Proposition 3.1 there exist $k_{1}, k_{2} \in K$ such that $k_{1} \cdot x, k_{2} \cdot y \in \mathfrak{b}$. Choose continuous maps $u, v:[0,1] \rightarrow K$ such that $u(1)=k_{1}$, $v(1)=k_{2}$, and $u(0)=v(0)=e$ (the identity element of $\left.K\right)$. Since

$$
f\left(v(r)^{-1} \exp \left(i s H_{m}\right) u(r) \cdot x\right)=\varphi\left(\exp \left(i s H_{m}\right) u(r) \cdot x, v(r) \cdot y\right),
$$

the point $f\left(v(r)^{-1} \exp \left(i s H_{m}\right) u(r) \cdot x\right)$ for fixed $r$ and variable $s$ traces an ellipse $\mathcal{E}_{r}$ in the complex plane (which may be degenerate). Since $u(0)=v(0)=e$, we have $\mathcal{E}_{0}=\mathcal{E}$. For $r=1$ we have

$$
f\left(v(1)^{-1} \exp \left(i s H_{m}\right) u(1) \cdot x\right)=\varphi\left(\exp \left(i s H_{m}\right) k_{1} \cdot x, k_{2} \cdot y\right) .
$$

Since $k_{1} \cdot x, k_{2} \cdot y \in \mathfrak{b}$, the above expression is independent of $s$, i.e., the "ellipse" $\mathcal{E}_{1}$ is just a point. Since $f\left(L_{m}(x)\right)$ is inside the ellipse $\mathcal{E}_{0}=\mathcal{E}$, there exists $r_{0} \in[0,1)$ such that $f\left(L_{m}(x)\right)$ lies on $\mathcal{E}_{r_{0}}$. This proves our claim.

Since $x \in \mathfrak{h}^{\perp}$, we have $t x=L_{1} L_{2} \cdots L_{l}(x)$ and our claim implies that $t x \leq x$.

Now assume that $\mathfrak{g}$ is of type $E_{6}$. The proof in this case is similar to the one above but requires some modifications.

Denote by $\Sigma$ the collection of closed root subsystems of $R$ of type $D_{5}$. As $|W|=2^{7} \cdot 3^{4} \cdot 5$ and the Weyl group of $D_{5}$ has order $2^{4} \cdot 5 !=2^{7} \cdot 3 \cdot 5$, it follows that $|\Sigma|=3^{3}$. For $\alpha \in R$ let $m$ be the number of subsystems $S \in \Sigma$ not containing $\alpha$. Clearly $m$ does not depend on $\alpha$, and so $m|R|=2^{5}|\Sigma|$ because $|R|-|S|=2^{5}$ for $S \in \Sigma$. It follows that $m=12$.

For a fixed $S \in \Sigma$ there exist exactly two elements $h \in \mathfrak{h}$ such that $\alpha(h)=0$ for all $\alpha \in S$ and $\{\alpha(h): \alpha \in R\}=\{0, \pm 1\}$. If $h$ is one of these two elements, then $-h$ is the other one. We choose one of these two elements and denote it by $h_{S}$. Let $\Gamma=\left\{h_{S}: S \in \Sigma\right\}$ and for $h \in \Gamma$ let

$$
R_{h}(k)=\{\alpha \in R: \alpha(h)=k\}, \quad k \in\{0, \pm 1\} .
$$

Then $R_{h}(0) \in \Sigma$ and each of the subsets $R_{h}( \pm 1)$ has cardinality 16 .

Let $L_{h}$ be the linear operator on $\mathfrak{g}$ which fixes the elements of $\mathfrak{h}$ and those of the root spaces $\mathfrak{g}^{\alpha}$ for $\alpha \in R_{h}(0)$ and on the other root spaces acts as multiplication by the scalar $t^{1 / 12}$. Thus we have

$$
L_{h}(x)=\sum_{\alpha \in R_{h}(0)} x_{\alpha}+t^{1 / 12} \sum_{\alpha \in R_{h}( \pm 1)} x_{\alpha} .
$$

We claim that $L_{h}(x) \leq x$. The proof of this claim is the same as in the case of root systems of type $D_{l}$ and we omit it. We just point out that $H_{m}$ should be 
replaced by $h(\in \Gamma), L_{h}$ should play the role of $L_{m}$, and $\sqrt{t}$ has to be replaced by $t^{1 / 12}$.

Since $\left\{L_{h}: h \in \Gamma\right\}$ is a commuting set of operators and for each $\alpha \in R$ there are exactly 12 elements $h \in \Gamma$ such that $\alpha \notin R_{h}(0)$, we obtain that

$$
\left(\prod_{h \in \Gamma} L_{h}\right)(x)=t x .
$$

Hence our claim implies that $t x \leq x$.

If $\mathfrak{g}$ is of type $E_{7}$ the argument is similar and we omit the details. We mention only that one should take $\Sigma$ to be the set of closed root subsystems of $R$ of type $E_{6}$.

As the Conjecture 3.9 is still open, it is of interest to ask whether or not $0 \leq x$ for all $x \in \mathfrak{g}$. We address this question in the following proposition.

Proposition 3.12. The following three statements are equivalent to each other:

(i) $\forall x \in \mathfrak{g}, 0 \leq x$;

(ii) $\forall f \in \mathfrak{g}^{*}, \forall x \in \mathfrak{g}, \operatorname{ker}(f)$ meets $K \cdot x$;

(iii) $\forall f \in \mathfrak{g}^{*}, \mathfrak{g}=\bigcup_{a \in K} \operatorname{ker}(a \cdot f)$.

If $\mathfrak{g}$ has no components of type $E_{8}, F_{4}$, or $G_{2}$, then these statements hold.

Proof. The equivalence of (i) and (ii) is immediate from the definition of the partial order " $\leq$ ". The equivalence of (ii) and (iii) follows from $(a \cdot f)(x)=f\left(a^{-1} \cdot x\right)$, where $a \in K, f \in \mathfrak{g}^{*}$, and $x \in \mathfrak{g}$. The first assertion is proved.

We now prove the second assertion. In view of Lemma 3.10 we may assume that $\mathfrak{g}$ is simple. By Theorem 3.11 we may exclude the cases $A_{l}, D_{l}, E_{6}$, and $E_{7}$. It remains to consider the cases $B_{l}$ and $C_{l}$.

Let $\mathfrak{g}$ be of type $B_{l}$. Again we shall make use of notations from [2, Chapitre VIII, $\S 13$, No. 2]. In this case

$$
R=\left\{ \pm \varepsilon_{i} \pm \varepsilon_{j}: i \neq j, 1 \leq i, j \leq l\right\} \cup\left\{ \pm \varepsilon_{i}: 1 \leq i \leq l\right\} .
$$

The coroot of $\varepsilon_{i}$ is the operator $2 H_{i}$. Let $\Sigma$ be the collection of all closed root subsystems $S$ of type $B_{l-1}$ (if $l=2$ we require that $S$ consists of two short roots). Define the subsets $R_{m}(k)$, for $1 \leq m \leq l, k=0, \pm 1$, by (3.2). Fix $t \in[0,1)$ and define the linear operators $L_{m}: \mathfrak{g} \rightarrow \mathfrak{g}$ as in the proof of Theorem 3.11. As in that proof, one can show that $L_{m}(x) \leq x$. The difference is that now, for $x$ given by (3.1), we obtain that

$$
L_{1} L_{2} \cdots L_{l}(x)=\sqrt{t} \sum_{\alpha \text { short }} x_{\alpha}+t \sum_{\alpha \text { long }} x_{\alpha} \leq x, \quad t \in[0,1) .
$$

In the special case $t=0$, we obtain that $0 \leq x$. it.

The proof when $\mathfrak{g}$ is of type $C_{l}$ is similar to that for the type $B_{l}$, and we omit

Remark 3.13. Assume that $\mathfrak{g}$ is simple, and express the highest root as a linear combination of $\Pi$. All coefficients in this linear combination are positive integers. The exceptional cases $E_{8}, F_{4}$, and $G_{2}$ are characterized by the property that all these coefficients are $\geq 2$.

An element $x \in \mathfrak{g}$ is said to be normal if $[x, \theta(x)]=0$. When $\mathfrak{g}=\mathfrak{s l}(n, \mathbf{C})$ and $\mathfrak{k}=\mathfrak{s u}(n)$, it reduces to the usual notion of normality of a matrix. 
Lemma 3.14. The element $x \in \mathfrak{g}$ is normal if and only if $K \cdot x$ meets $\mathfrak{h}$.

Proof. Let $x$ be normal and write $x=y+i z$ where $y, z \in \mathfrak{k}$. Then $\theta(x)=y-i z$ and so $[y, z]=0$. The assertion now follows from the fact that maximal abelian subalgebras of $\mathfrak{k}$ are its Cartan subalgebras and the latter are all $K$-conjugate. The converse is obvious.

Since the maximal tori of $K$ are conjugate, the normality of $x \in \mathfrak{g}$ and Lemma 3.14 are independent of the choice of $\mathfrak{t}$ and thus of $\mathfrak{h}$. It is evident from the definition.

The following proposition is useful, although it is an immediate consequence of the definitions.

Proposition 3.15. Let $\mathfrak{k}_{1}$ be a semisimple subalgebra of $\mathfrak{k}$ and $\mathfrak{g}_{1}=\mathfrak{k}_{1}+i \mathfrak{k}_{1}$ its complexification. If $x, y \in \mathfrak{g}_{1}$ and $x \leq y$ in $\mathfrak{g}_{1}$, then also $x \leq y$ in $\mathfrak{g}$.

Proof. If $f \in \mathfrak{g}^{*}$ then $f \mid \mathfrak{g}_{1} \in \mathfrak{g}_{1}^{*}$ and so $f(x) \in f\left(K_{1} \cdot y\right)$, where $K_{1}$ is the connected subgroup of $K$ with Lie algebra $\mathfrak{k}_{1}$. Hence $f(x) \in f(K \cdot y)$ for all $f \in \mathfrak{g}^{*}$, i.e., $x \leq y$ is valid also in $\mathfrak{g}$.

We can now show that Conjecture 3.9 is true for normal elements.

Proposition 3.16. If $x$ is normal, then $t x \leq x$ for all $t \in[0,1]$.

Proof. Since $x$ is normal, by Lemma 3.14, we may assume that $x \in \mathfrak{h}$. Let $S$ be as in Lemma 3.3 and let $\mathfrak{s}$ be its Lie algebra. Since $S$ is $\theta$-stable, $\mathfrak{k}_{1}=\mathfrak{s} \cap \mathfrak{k}$ is a compact real form of $\mathfrak{s}$. Since all simple components of $\mathfrak{s}$ are of type $A$ and $x \in \mathfrak{h} \subseteq \mathfrak{s}$, Theorem 3.11 shows that $t x \leq x$ in $\mathfrak{s}$ for all $t \in[0,1]$. It remains to apply Proposition 3.15 .

\section{THE CASE $\mathfrak{g}=\mathfrak{s l}_{2}$}

In this section it will be understood that $G=\mathrm{SL}(2, \mathbf{C}), K=\mathrm{SU}(2), \mathfrak{g}=\mathfrak{s l}(2, \mathbf{C})$, and $\mathfrak{k}=\mathfrak{s u}(2)$. We also set

$$
x=\left(\begin{array}{cc}
x_{11} & x_{12} \\
x_{21} & -x_{11}
\end{array}\right) \in \mathfrak{g} .
$$

Define the $K$-invariant polynomial functions $f_{1}: \mathfrak{g} \rightarrow \mathbf{C}$ and $f_{2}: \mathfrak{g} \rightarrow \mathbf{R}$ by

$$
f_{1}(x)=\frac{1}{2} \operatorname{tr}\left(x^{2}\right), \quad f_{2}(x)=\frac{1}{2} \operatorname{tr}\left(x x^{*}\right) .
$$

Explicitly, we have

$$
f_{1}(x)=x_{11}^{2}+x_{12} x_{21}, \quad f_{2}(x)=\left|x_{11}\right|^{2}+\frac{1}{2}\left(\left|x_{12}\right|^{2}+\left|x_{21}\right|^{2}\right) .
$$

Since $\left|f_{1}(x)\right| \leq f_{2}(x)$, the point $\left(f_{1}(x), f_{2}(x)\right)$ belongs to the closed convex cone

$$
C=\{(z, t) \in \mathbf{C} \times \mathbf{R}:|z| \leq t\} .
$$

Define the continuous map $F: \mathfrak{g} \rightarrow C$ by $F(x)=\left(f_{1}(x), f_{2}(x)\right)$. It is well known that the invariants $f_{1}$ and $f_{2}$ separate the $K$-orbits in $\mathfrak{g}$, i.e., two matrices $x, y \in \mathfrak{g}$ belong to the same $K$-orbit if and only if $F(x)=F(y)$. This is an old result of F.D. Murnaghan [8] (see also [10, Corollary 2.35]).

Let us define the closed subset $S$ of $\mathfrak{g}$ by:

$$
S=\left\{w\left(\begin{array}{cc}
0 & 1 \\
\lambda & 0
\end{array}\right): w \in \mathbf{C}, \lambda \in[0,1]\right\} .
$$


Lemma 4.1. $F(S)=F(\mathfrak{g})=C$. Consequently, every $K$-orbit in $\mathfrak{g}$ meets $S$ i.e., $\mathfrak{g}=K \cdot S$.

Proof. In order to prove this assertion, it suffices to show that for a given point $(z, t) \in C$ there exist $w \in \mathbf{C}$ and $\lambda \in[0,1]$ such that

$$
\lambda w^{2}=z, \quad\left(1+\lambda^{2}\right)|w|^{2}=2 t .
$$

If $t=0$ then also $z=0$ and we can take $w=0$ and $\lambda=0$. If $t>0$ and $z=0$, we can take $\lambda=0$ and $w=\sqrt{2 t}$. Finally let $z \neq 0$. Then the second equation above can be replaced by

$$
\left(1+\lambda^{2}\right)|z|=2 \lambda t .
$$

This equation has a unique solution for $\lambda$ in the interval $(0,1]$. After that we can solve the equation $\lambda w^{2}=z$ for $w$.

We equip the orbit space $\mathfrak{g} / K$ with the quotient topology and denote by $\pi: \mathfrak{g} \rightarrow$ $\mathfrak{g} / K$ the projection map. The map $F_{0}: \mathfrak{g} / K \rightarrow C$ induced by $F$ is a continuous bijection. We shall prove that it is in fact a homeomorphism.

Proposition 4.2. $F_{0}: \mathfrak{g} / K \rightarrow C$ is a homeomorphism.

Proof. It suffices to show that $F$ is a proper map, i.e., if $X \subseteq C$ is compact, then $F^{-1}(X)$ is also compact. Choose $t_{0} \geq 0$ such that $t \leq t_{0}$ for all points $(z, t) \in X$. Then $f_{2}(x) \leq t_{0}$ for all matrices $x \in F^{-1}(X)$. Hence $F^{-1}(X)$ is a closed and bounded subset of $\mathfrak{g}$, and so it is compact.

The closed subset $S$ fails to be a section of the map $F: \mathfrak{g} \rightarrow C$ since, for $x \in S \backslash\{0\}$, the intersection $S \cap K \cdot x$ is $\{ \pm x\}$ if $x^{2} \neq 0$, and $\left\{e^{i \theta} x: \theta \in \mathbf{R}\right\}$ if $x^{2}=0$. However, it will be convenient to use the elements of $S$ as representatives of the $K$-orbits in $\mathfrak{g}$, taking into account the ambiguities just mentioned.

We now discuss the partial order " $\leq$ ". The following theorem is a special case of a result of Nakazato [9] (see [7] for another proof).

Theorem 4.3. Let $\mathfrak{g}=\mathfrak{s l}(2, \mathbf{C}), K=\mathrm{SU}(2)$, and

$$
a=\left(\begin{array}{cc}
0 & a_{12} \\
a_{21} & 0
\end{array}\right), \quad b=\left(\begin{array}{cc}
0 & b_{12} \\
b_{21} & 0
\end{array}\right),
$$

with $a_{12} \geq a_{21} \geq 0$ and $b_{12} \geq b_{21} \geq 0$. If $f \in \mathfrak{g}^{*}$ is defined by $f(x)=\operatorname{tr}(a x)$, then $f(K \cdot b)$ is the elliptical disk in the complex plane in standard position with vertices $\pm\left(a_{12} b_{12}+a_{21} b_{21}\right)$ and $\pm\left(a_{12} b_{12}-a_{21} b_{21}\right) i$.

In order to make the order " $\leq$ " useful, one needs a simple test for $x \leq y$ to hold. Unfortunately, we were not able to find such a test for arbitrary $x$ and $y$. By using the above theorem, we can handle some particular cases. The proofs are straightforward and are omitted.

Lemma 4.4. Let

$$
x=w\left(\begin{array}{ll}
0 & 1 \\
\lambda & 0
\end{array}\right), \quad w \in \mathbf{C}, \lambda \in[0,1] .
$$

If $b=\left(\begin{array}{ll}0 & 1 \\ 0 & 0\end{array}\right)$ then

$$
\begin{aligned}
& x \leq b \Longleftrightarrow(1+\lambda)|w| \leq 1, \\
& b \leq x \Longleftrightarrow(1-\lambda)|w| \geq 1 .
\end{aligned}
$$


If $b=\left(\begin{array}{ll}0 & 1 \\ 1 & 0\end{array}\right)$ then

$$
x \leq b \Longleftrightarrow \lambda=1, w \in \mathbf{R},|w| \leq 1 .
$$

\section{REFERENCES}

[1] N. Bourbaki, Groupes et algèbres de Lie, Chapitres 4, 5 et 6, Hermann, Paris, 1968.

[2] N. Bourbaki, Groupes et algèbres de Lie, Chapitres 7 et 8, Hermann, Paris, 1975.

[3] W.S. Cheung and N.K. Tsing, The C-numerical range of matrices is star-shaped, Linear and Multilinear Algebra 41 (1996), 245-250.

[4] S. Helgason, Differential Geometry, Lie Groups, and Symmetric Spaces, Academic Press, New York, 1978.

[5] R.A. Horn and C.R. Johnson, Topics in Matrix Analysis, Cambridge Univ. Press, 1991.

[6] J.E. Humphreys, Introduction to Lie Algebras and Representation Theory, Springer-Verlag, New York 1972.

[7] C.K. Li, Some convexity theorems for the generalized numerical ranges, Linear and Multilinear Algebra, 40 (1996), 235-240.

[8] F.D. Murnaghan, On the unitary invariants of a square matrix, Anais Acad. Brasil. Cienc., 26 (1954), 1-7.

[9] H. Nakazato, The C-numerical range of a $2 \times 2$ matrix, Sci. Rep. Hirosaki Univ., 41 (1994), 197-206.

[10] K.S. Sibirskiii, The Algebraic Invariants of Differential Equations and Matrices (in Russian), Stiintsa, Kishinev, 1976.

[11] T.Y. Tam, On the shape of numerical range associated with Lie groups, Taiwanese J. Math. 5 (2001), 497-506.

Department of Pure Mathematics, University of Waterloo, Waterloo, Ontario, N2L 3G1, CANADA

Department of Mathematics, Auburn University, AL 36849-5310, USA

E-mail address: djokovic@uwaterloo.ca

E-mail address: tamtiny@auburn.edu 